ARTICLE

\title{
Cornelia de Lange syndrome-associated mutations cause a DNA damage signalling and repair defect
}

Gabrielle Olley¹, Madapura M. Pradeepa (1) 1,2, Graeme R. Grimes', Sandra Piquet ${ }^{3}$, Sophie E. Polo (1) ${ }^{3}$, David R. FitzPatrick', Wendy A. Bickmore (iD ${ }^{1 凶}$ \& Charlene Boumendil (1) 1,4凶

Cornelia de Lange syndrome is a multisystem developmental disorder typically caused by mutations in the gene encoding the cohesin loader NIPBL. The associated phenotype is generally assumed to be the consequence of aberrant transcriptional regulation. Recently, we identified a missense mutation in BRD4 associated with a Cornelia de Lange-like syndrome that reduces BRD4 binding to acetylated histones. Here we show that, although this mutation reduces BRD4-occupancy at enhancers it does not affect transcription of the pluripotency network in mouse embryonic stem cells. Rather, it delays the cell cycle, increases DNA damage signalling, and perturbs regulation of DNA repair in mutant cells. This uncovers a role for BRD4 in DNA repair pathway choice. Furthermore, we find evidence of a similar increase in DNA damage signalling in cells derived from NIPBL-deficient individuals, suggesting that defective DNA damage signalling and repair is also a feature of typical Cornelia de Lange syndrome.

\footnotetext{
${ }^{1}$ MRC Human Genetics Unit, Institute of Genetics and Cancer, University of Edinburgh, Crewe Road, Edinburgh, UK. ${ }^{2}$ Blizard institute, Barts and The London School of Medicine and Dentistry, Queen Mary University of London, London, UK. ${ }^{3}$ Epigenetics and Cell Fate Centre, UMR7216 CNRS, Université de Paris,

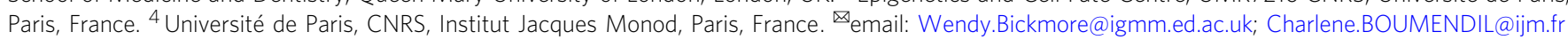


C ornelia de Lange syndrome (CdLS) is a clinically distinctive neurodevelopmental disorder (OMIM:122470). Disease severity varies greatly and patients can suffer from a range of symptoms including: a characteristic facial appearance, upper limb abnormalities, intellectual disability and delayed growth ${ }^{1}$. CdLS is described as a 'cohesinopathy'l-most cases can be attributed to heterozygous loss of function mutation in NIPBL encoding a protein involved in loading of the cohesin complex onto chromatin ${ }^{2}$. Mutation in genes encoding cohesin complex proteins SMC1, SMC3 and RAD21, or HDAC8 (SMC3 deacetylase), have also been identified in CdLS-like probands ${ }^{2}$. However cells from CdLS patients have no obvious defects in sister chromatid cohesion ${ }^{3}$, and individuals with mutations in SMC1, SMC3 and $R A D 21$ are often considered 'atypical' in terms of facial appearance and growth, and are less likely to have limb defects than those with NIPBL mutations ${ }^{4}$.

Dysregulated gene expression has been proposed to be the main mechanism underlying $\mathrm{CDLS}^{5,6}$. Mutations in genes encoding chromatin regulators unrelated to cohesin, such as ANKRD11, KMT2A, AFF4 and the bromodomain and extraterminal domain (BET) protein $\mathrm{BRD} 4$, have been reported to cause CdLS-like phenotypes ${ }^{1}$ suggesting that chromatin dysregulation may play a role in CdLS as well. Additionally, increased sensitivity to DNA damage has been reported in CdLS patient cells ${ }^{7}$, but the mechanism underlying this defect is unknown and its participation in the disease aetiology remains unclear.

Recently, we described de novo deletion and missense mutations in BRD4 associated with a clinical phenotype overlapping $\mathrm{CdLS}^{8}$. BRD4 binds acetylated lysine residues in histones $\mathrm{H} 3$ and $\mathrm{H} 4$ through its two N-terminal bromodomain domains (BD). BRD4 localises to promoters and enhancers of active genes and is particularly enriched at super enhancers (SEs) ${ }^{9,10}$. BRD4 is a key regulator of transcription; through its C-terminal domain it recruits positive transcription elongation factor $(\mathrm{P}-\mathrm{TEFb})$ and the Mediator complex to promoters and enhancers, whilst its extraterminal domain confers transcriptional activation through the recruitment of CHD4, JMJD6 and NSD3 ${ }^{11,12}$.

The CdLS-associated BRD4 missense mutation is in the second bromodomain (BD2) (NM_058243.2:c.1289A > G, p.(Tyr430Cys), termed here as Y430C (Fig. 1a), and results in decreased binding to acetylated histones ${ }^{8}$. To gain further insights into the mechanisms underlying CdLS, and the role of BRD4, we investigated the phenotype of mouse embryonic stem cells (mESCs) homozygous for the orthologous amino acid substitution in mouse Brd4 (actually p.Tyr431Cys but for simplicity here termed $B r d 4^{Y 430 C}$ ). Here we show that the decreased affinity for acetylated lysines results in diminished occupancy of BRD4 ${ }^{\mathrm{Y} 430 \mathrm{C}}$ at cis regulatory elements (CREs) across the genome, including the super-enhancers of pluripotency genes. However, we find no evidence of altered transcription of the pluripotency network in these cells. Instead, we report increased and more persistent DNA damage signalling and cell cycle checkpoint activation in Brd4 ${ }^{\text {Y430C }}$ mESCs. We show increased persistent foci of the DNA damage response (DDR) protein 53BP1 upon double-strand break (DSB) induction in Brd4 mutant cells. 53BP1 is a key factor in the regulation of DNA repair pathway choice that inhibits repair by homologous recombination (HR). We also show increased foci of the downstream effectors of 53BP1, Rif1 and the Mad2l2 (Rev7) subunit of the shieldin complex in the mutant cells $^{13-22}$ and decreased recruitment of RAD51, suggesting impaired HR repair. Further, we show that cells from CdLS patients harbouring mutations in NIPBL have a similar DDR phenotype, indicating there may be a previously underappreciated role for the DNA damage response in the aetiology of CdLS.

\section{Results}

Reduced occupancy of Y430C-BRD4 at cis-regulatory elements. Our previous work suggested that the Y430C mutation abrogates BRD4 binding to acetylated histones in vitro and in vivo. To determine the genome-wide effect of this reduced affinity we carried out BRD4 ChIP-seq in two independently generated mESCs lines engineered by CRISPR-Cas9 to carry the Y430C mutation on both alleles of Brd4. Immunoblotting showed that the Y430C mutant BRD4 protein was present at levels equivalent to that of BRD4 in wild-type cells (Supplementary Fig. S1a) and was efficiently immunopreciptated by anti-Brd4 antibodies (Supplementary Fig. S1b). As expected, BRD4 was enriched over CREs (SEs, typical enhancers and promoters) in both wild-type (WT) and Y430C cells (Fig. 1b, Supplementary Fig. S1c). However, consistent with a lowered affinity for acetyl-lysines, there was a general decrease in BRD4 occupancy in both clones of Y430C cells, most striking at enhancers and super-enhancers (SE) (Fig. 1c, d, Supplementary Fig. S1c-d). In mESCs, BRD4 binding to SEs regulates the transcription of stem cell identity genes ${ }^{9}$. As BRD4 ${ }^{\mathrm{Y} 430 \mathrm{C}}$ occupancy is decreased at the SEs of a number of stem cell identity genes, this suggests that there might be decreased transcription of these genes in mutant cells.

Decreased occupancy of mutant BRD4 at CREs does not have major effects on transcription in mESCs. The use of inhibitors that competitively bind the acetyl-lysine binding pockets of BET proteins has shown that loss of BRD4 binding disrupts the expression of target genes, especially genes regulated by SEs ${ }^{10}$. Consistent with this, we observed decreased expression of the SE associated genes Nanog, Myc, Klf4 and Oct4 in both WT and Y430C mESCs after treatment with JQ1 (Fig. 2a). However, we did not observe any decrease in levels of Klf4, Nanog and Oct4 mRNAs in Y430C compared to WT mESCs by RT-qPCR (Fig. 2b).

To determine whether mRNA stability was masking an effect on transcription per se, we performed 4-thiouridine sequencing (4SU-seq) to assay nascent transcription. Transcription was surprisingly similar between WT and Y430C mESCs (Pearson correlation coefficient $=0.98$ ) (Fig. $2 \mathrm{c}$, d and data from an independent Y430C in Supplementary Fig. 2a). In particular, decreased BRD4 binding at SEs did not lead to transcriptional changes at stem cell identity genes (Fig. 2c-e, replicate in Supplementary Fig. 2b), or of eRNAs at the SEs themselves (Fig. 2f, Supplementary Fig. 2c). Due to normalisation, these experiments could not rule out that transcription is not globally decreased in the mutant ESCs. We therefore performed a spike-in RNA-seq experiment, using RNA from Drosophila cells for normalisation. Again, we did not observe any major transcriptional differences between WT and Y430C cells (Supplementary Fig. 2d, e). We conclude that the decreased occupancy of $\mathrm{BRD} 4^{\mathrm{Y} 430 \mathrm{C}}$ at CREs in mESCs is not sufficient to affect the transcription of associated genes.

This result is surprising, given BRD4's well-documented roles in transcriptional regulation. Therefore, we also analysed gene expression in embryonic fibroblasts (MEFs) that had been derived from either wild-type or $\mathrm{Brd} 44^{Y 430 C}$ homozygous embryos ${ }^{8}$. In contrast to homozygous null Brd4 embryos, which show significant growth retardation at E13.5 of embryonic development, $B r d 4^{Y 430 C}$ homozygous embryos are reported to be morphologically indistinguishable from wild-type at this stage 8 suggesting the absence of major developmental gene dysregulation. However, analysis of gene expression shows significant alteration of gene expression between wild-type and mutant MEFS (Supplementary Fig. 3). We consider that this difference in the transcriptional consequences of Brd4 ${ }^{\mathrm{Y} 430 \mathrm{C}}$ between ESCs and 
a

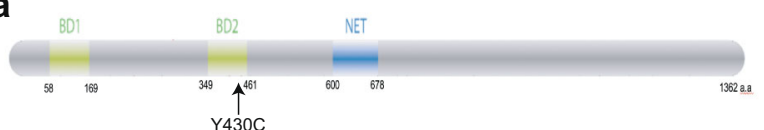

b
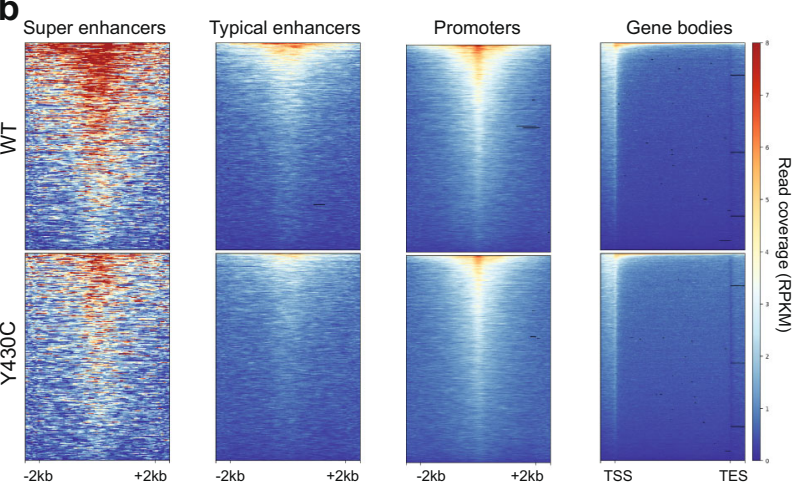

d

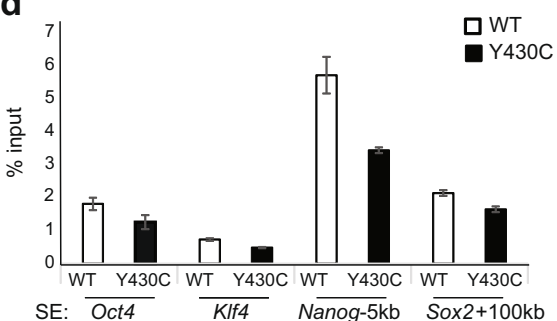

C

C
苟
$\frac{0}{0}$
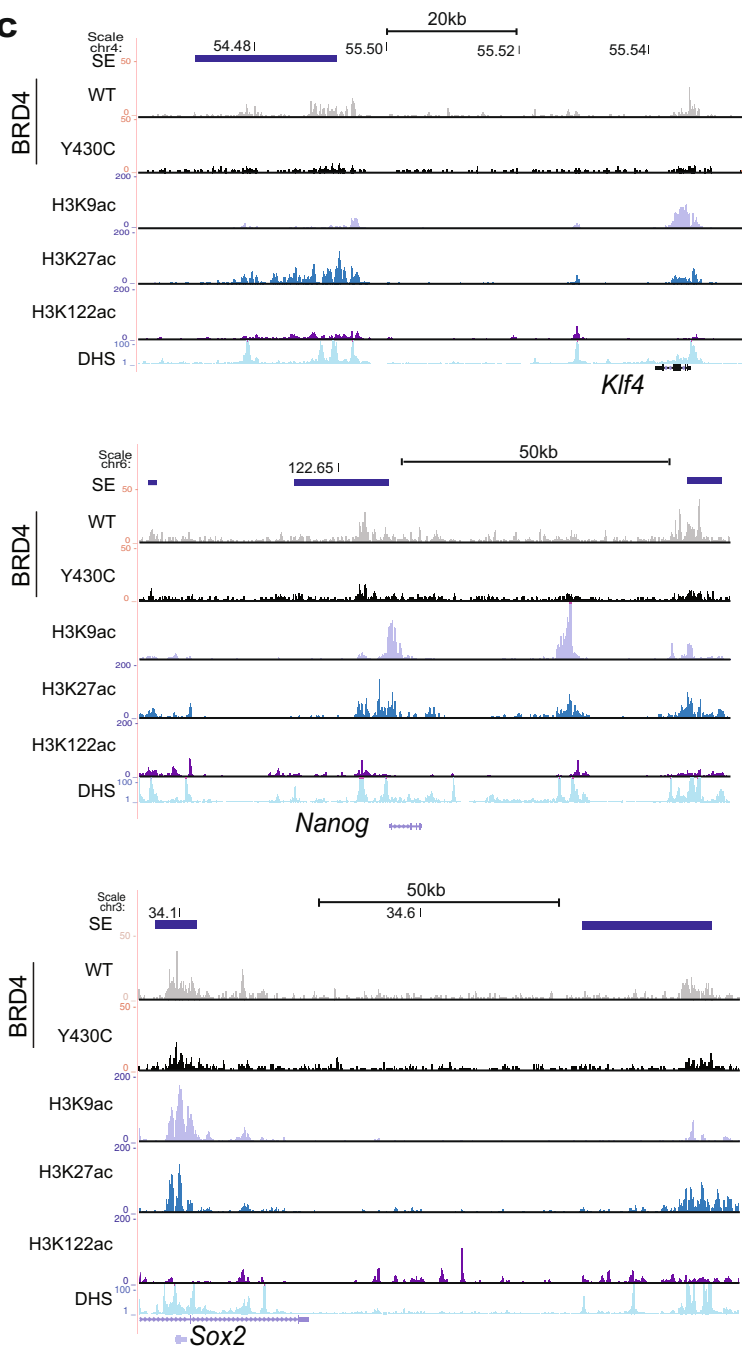

Fig. 1 Decreased binding of BRD4 at CREs in Y430C mESCs. a Cartoon of BRD4 showing location of the Y430C mutation in the second bromodomain (BD2). b Heatmaps show enrichment of wild-type (WT) and Y430C BRD4 ChIP over super enhancers (SE), typical enhancers, promoters and gene bodies. c UCSC genome browser screenshot showing reads per 10 million over the KIf4, extended Nanog and Sox2 loci for BRD4 ChIP-seq in WT and Y430C mESCs. Extent of SEs are shown in blue. Below are shown previously published ChIP-seq data for H3K27ac (ENCSR000CDE), H3K9ac (ENCSR000CGS), H3K122ac (GSE66023) and DNase I hypersensitivity (DHS). Genome co-ordinates (Mb) are from the mm9 assembly of the mouse genome. Biological replicate from an independent $Y 430 \mathrm{C}$ clone are in Supplementary Fig. 1. d ChIP-qPCR measuring concentration of BRD4 ChIP DNA relative to input across the SEs of Oct4, Klf4, Nanog, and Sox2; in WT and Y430C mESCs. Data are represented as mean \pm SEM from 3 technical replicates.

MEFS may reflect the differing dependencies on epigenetic regulators at different stages of development.

Y430C-BRD4 mESCs have a delayed cell cycle and increased cell cycle checkpoint activation. We noted that $B R D 4^{Y 430 C}$ mESCs grew slower and showed an accumulation of cells in G2/ M (33.7\%), compared to their WT counterparts (27.8\%) (Fig. 3a, b, Supplementary Fig. 4a and Supplementary Fig. 9). This observation, together with the recently reported roles for BRD4 in the DDR and DNA repair ${ }^{23-26}$ led us to investigate potential DDR defects in mutant cells.

The DDR allows coordination between DNA repair and cell cycle progression. Recognition of DNA damage by sensor proteins initiates a cascade that results in the phosphorylation and activation of the checkpoint kinases CHK1 and CHK2, delaying or blocking cell cycle progression ${ }^{27}$. $\mathrm{CHK} 1$ is the main kinase required for delay at $\mathrm{G} 2 / \mathrm{M}^{27}$. To determine whether the altered cell cycle in $B R D 4^{Y 430 C}$ cells is associated with increased activation of the G2/M checkpoint, we analysed CHK1 phosphorylation (CHK1-P) after treatment with neocarzinostatin (NCS), a radiomimetic drug which induces mainly DSBs. CHK1$\mathrm{P}$ is increased in both WT and Y430C mESCs cell lines $1 \mathrm{~h}$ post NCS treatment, which is resolved by $16 \mathrm{~h}$. However, the levels of CHK1-P are higher in BRD4 ${ }^{Y 430 C}$ mESCs (Fig. 3c, Supplementary Fig. $4 \mathrm{~b}$ ), suggesting an increased checkpoint activation.

These results suggest a defect in DNA repair or signalling caused by $\mathrm{BRD} 4^{\mathrm{Y} 430 \mathrm{C}}$. BRD4 has been shown to be directly involved in DNA repair through the transcriptional regulation of DNA repair proteins $24,25,28$. However, 4SU-seq showed that transcription of genes encoding DNA repair proteins was unaffected in BRD $4^{Y 430 C}$ mESCs (Supplementary Fig. 4c, d) and immunoblotting showed that overall levels of 53BP1 and Rad51 were not significantly altered in the mutant cells (Supplementary Fig. 4e).

Y430C-BRD4 mESCs have increased DDR signalling. Although not being directly recruited to DSBs $^{29}$ (Supplementary Fig. 5a), $\mathrm{BRD} 4$ restricts the DDR and depletion of BRD4 isoform $\mathrm{B}$ leads to 
a

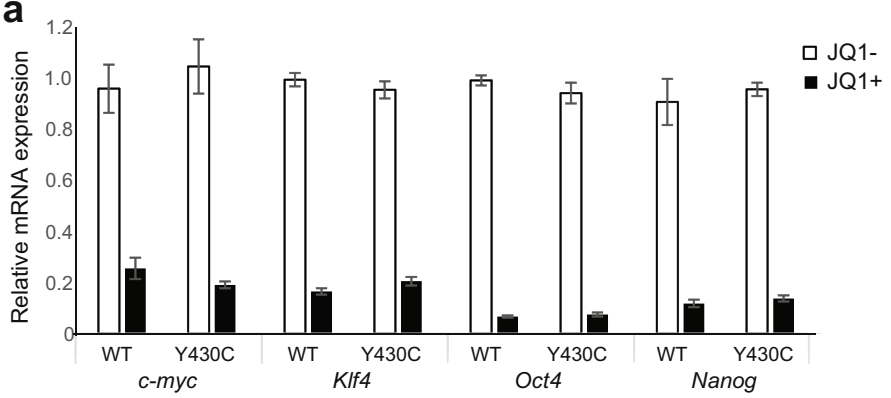

b

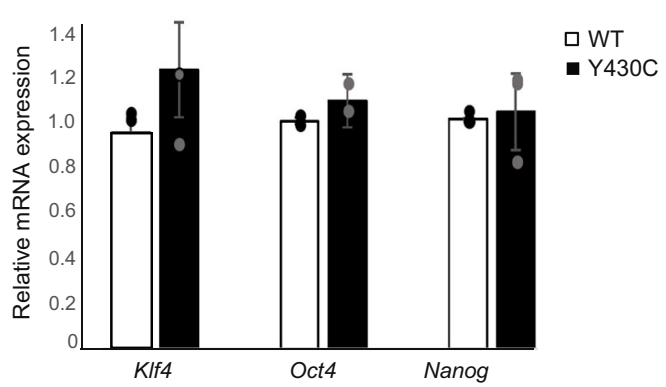

C

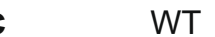

Y430C

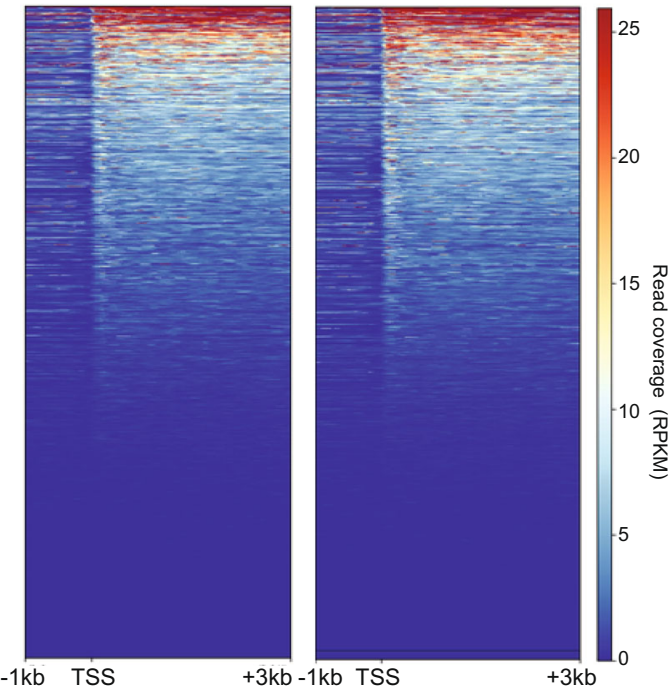

d

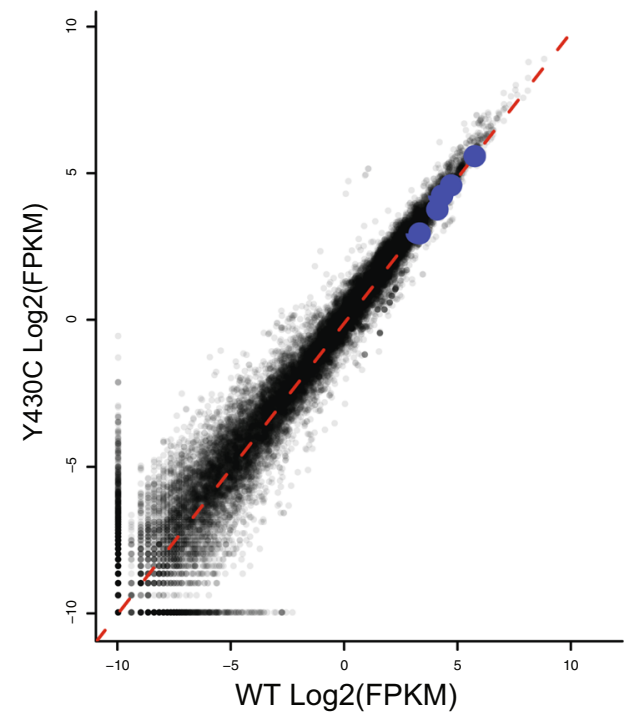

e
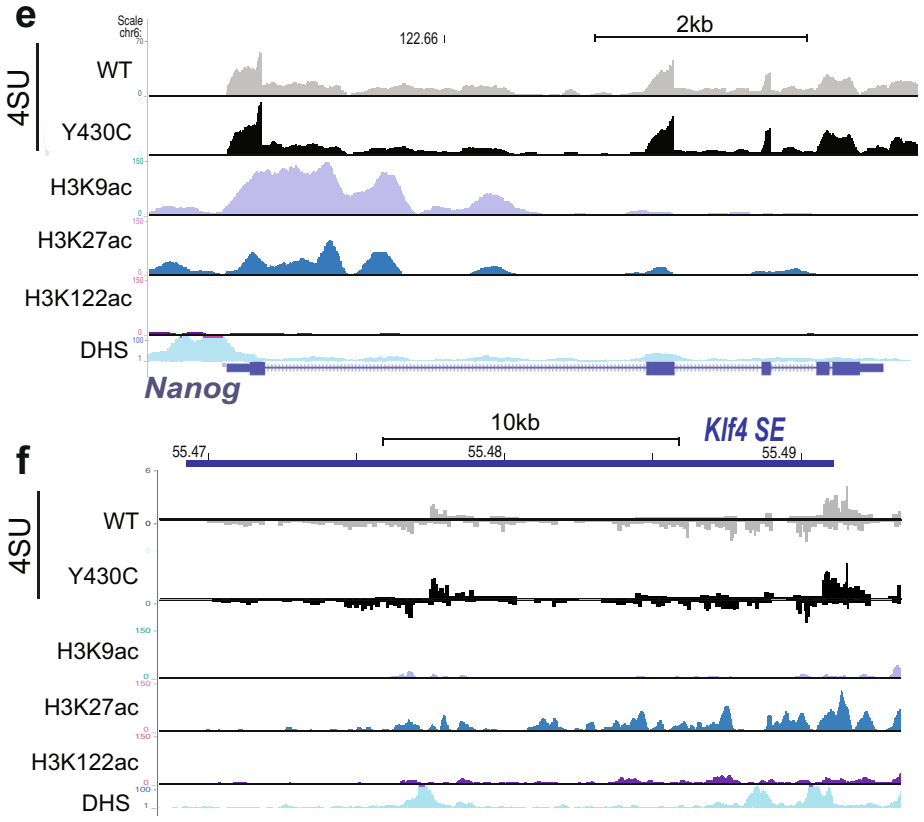

Fig. 2 Similar transcription in WT and Y430C mESCs. a RT-qPCR measuring mRNA of c-myc, Klf4, Oct4 and Nanog in mESCs after treatment with 300 $\mathrm{nM} \mathrm{JQ1} \mathrm{+,} \mathrm{relative} \mathrm{to} \mathrm{that} \mathrm{in} \mathrm{untreated} \mathrm{cells} \mathrm{(JQ1-).} \mathrm{Data} \mathrm{are} \mathrm{represented} \mathrm{as} \mathrm{mean} \pm$ SEM from 3 technical replicates. b RT-qPCR measuring mRNA for KIf4, Oct4 and Nanog in WT and Y $430 \mathrm{C}$ mESCs. mRNA concentration is shown relative to WT set at 1. Data are represented as mean \pm SEM from 3 biological replicates. c Heatmaps show enrichment of 4sU-seq data in WT and Y430C cells over transcribed regions ( $-1 \mathrm{~kb}$, TSS and +3 kb) (mm9_refseq). d Scatter plot of the 4sU-seq data in WT and Y430C cells, highlighting pluripotency genes in blue (Nanog, Sox2, KIf4, Esrrb, Pou5f1). Red dashed line shows best fitted line. Pearson correlation coefficient $=0.98$. e, $\mathbf{f}$ UCSC browser screenshot showing 4 SU-seg reads per 10 million over e the Nanog locus and $\mathbf{f}$ the KIf4 super-enhancer in WT and Y430C cells and ChIP-seq tracks for various histone modifications and DNasel hypersensitivity in WT cells. Genome coordinates $(\mathrm{Mb})$ are from the $\mathrm{mm} 9$ assembly of the mouse genome. Data from a biological replicate $\mathrm{Y} 430 \mathrm{C}$ clone are in Supplementary Fig. 2.

increased DDR signalling ${ }^{23}$. We therefore tested whether $\mathrm{BRD} 4{ }^{\mathrm{Y} 430 \mathrm{C}}$ affects DNA damage signalling. mESCs have constitutively high levels of $\gamma \mathrm{H} 2 \mathrm{AX}$, even in the absence of a DNA damaging stimulus ${ }^{30}$. We therefore used 53BP1 as a marker of DDR. 53BP1 is recruited to DSBs, spreads to form microscopically visible foci and acts as a scaffold for the recruitment of further DSB response proteins, to regulate the choice of DNA repair pathway and to promote cell cycle checkpoint signalling ${ }^{31}$.

Immunofluorescence showed formation of multiple 53BP1 foci, representing DNA damage sites, upon DSB induction (1 h 


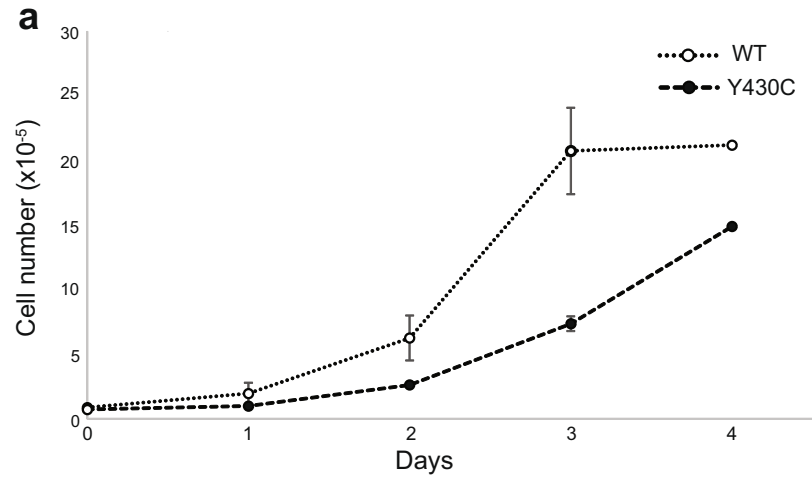

C
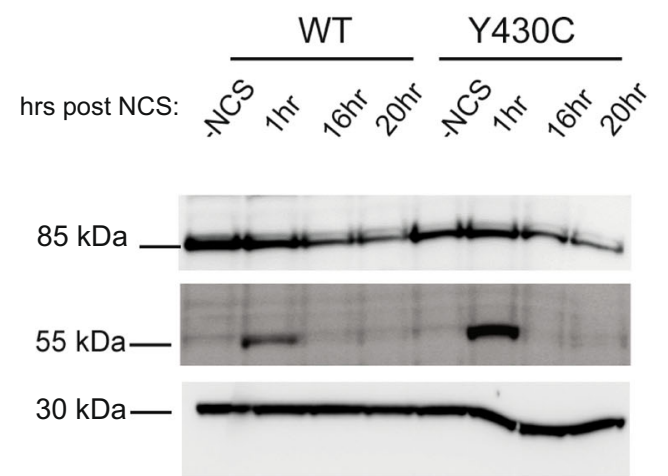

Chk1

Chk1-P

PCNA b

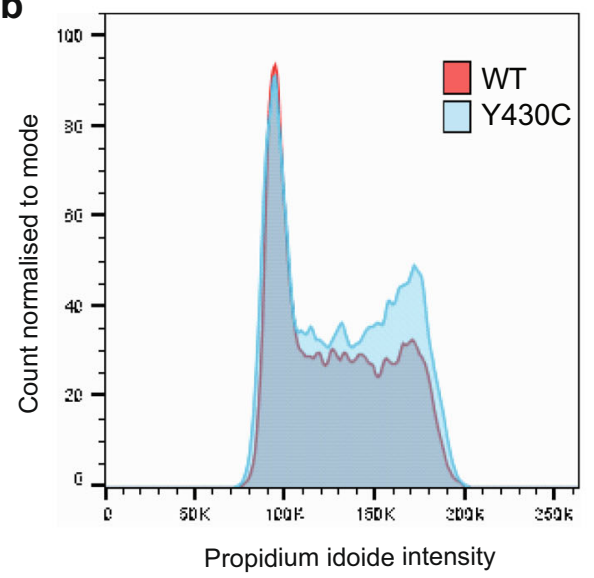

Fig. 3 Increased G2/M checkpoint activation in Y430C mESCs. a Graph shows average number of WT and Y $430 \mathrm{C}$ cells per well at 1, 2, 3 and 4 days post seeding. Data are represented as mean \pm SEM from 3 technical replicates. $\mathbf{b}$ Overlaid graphs show WT and Y $430 \mathrm{C}$ cell cycle profiles, as determined by flow cytometry. Graphs illustrate the cell count, which correlates to propidium iodide intensity. Biological replicate in Supplementary Fig. 4a. c Immunoblot using antibodies against Lamin B CHK1 and CHK1-P after treatment of WT and Y430C mESCs with NCS and for various times (hrs) of recovery (source data are provided as a Source Data file). Data from an independent experiment are presented in Supplementary Fig. 4.

after NCS treatment). These foci are only present at low levels prior to NCS treatment and decrease in number at 16 and $20 \mathrm{~h}$ post treatment, as cells repair the damage (Fig. 4a). Supporting the hypothesis that the Y430C mutation impairs the role of BRD4 in DDR restriction, we observed that 53BP1 foci are larger in $B R D 4^{Y 430 C}$ mESCs than in WT (Fig. 4a, b). In addition, whilst the number of 53BP1 foci in WT cells returns to pre-treatment levels at 16 and $20 \mathrm{~h}$ time points, the number of 53BP1 foci in $B R D 4^{Y 430 C}$ cells remains higher (Fig. $4 \mathrm{a}$, c, Supplementary Fig. 5b, c), suggesting that DNA repair itself could be impaired.

During our analysis of transcription, we found potential evidence for aneuploidy for chromosome 11 in $B R D 4^{Y 430 C}$ cells. Concerned that the DNA repair defects we observed in $B R D 4^{Y 430 C}$ cells were caused by aneuploidy rather than the BRD4 mutation, we repeated the 53BP1 staining using WT and $B R D 4^{Y 430 C}$ mESCs that had been confirmed to have a diploid karyotype. This analysis confirmed that the increased number and size of 53BP1 foci after NCS treatment is specific to cells carrying the $B R D 4^{Y 430 C}$ mutation, and not an additional chromosome 11 (Supplementary Fig. 6).

Given the accumulation of BRD4 ${ }^{Y 430 C}$ cells in S/G2 (Fig. 3), one possible origin of 53BP1 foci is the formation of Oct1/PTF/ transcription (OPT) domains arising as a consequence of problems encountered in S-phase and propagated through mitosis into the subsequent $\mathrm{G} 1^{32,33}$. Indeed inhibition of BRD4 by BET inhibitors has been reported to induce replication stress $^{34}$. However, immunofluorescence showed that 53BP1 foci present in $B R D 4^{Y 430 C}$ cells, either with or without NCS treatment, do not co-localise with OPT domains marked by Oct1 (Supplementary Fig. 7).

Defective DSB repair in Y430C-BRD4 cells. For the most part, DSBs are repaired by either non-homologous end-joining (NHEJ) or $\mathrm{HR}^{35}$. Use of the appropriate pathway is important for faithful repair and is determined by antagonistic recruitment of 53BP1 and BRCA1 ${ }^{31}$. 53BP1 inhibits DSB end resection, the initial step of HR, thereby promoting NHEJ and inhibiting HR. Downstream effectors of 53BP1 in the regulation of resection include RIF1 ${ }^{19-22}$ and the recently identified shieldin complex (SHLD1, SHLD2, SHLD3 and MAD2L2) ${ }^{13-18}$. If timely repair does not occur by NHEJ, BRCA1 promotes the release of RIF1, leading to endresection and $\mathrm{HR}$.

As BRD4 ${ }^{Y 430 C}$ mESCs show increased numbers and size of 53BP1 foci compared to WT cells, we reasoned that there may also be increased recruitment of the downstream effectors of 53BP1 such as RIF1 and MAD2L2. Indeed, we observed an increased number of RIF1 (Fig. 5a, b, Supplementary Fig. 5d, e) and MAD2L2 (Fig. 5c, d, Supplementary Fig. 5f, h) foci in $B R D 4^{Y 430 C}$ compared to WT cells at all time-points, similar to 53BP1. Conversely, we observed a significant decrease in the number of foci of RAD51, a protein necessary for HR repair, in mutant cells at $1 \mathrm{~h}$ post NCS (Fig. 6a, b, Supplementary Fig. 5h, i), suggesting a repression of HR. Given our cell cycle analysis (Fig. 3b) this cannot be simply explained by fewer cells in S/G2. Given the role of the shieldin complex in protecting DSB 
a
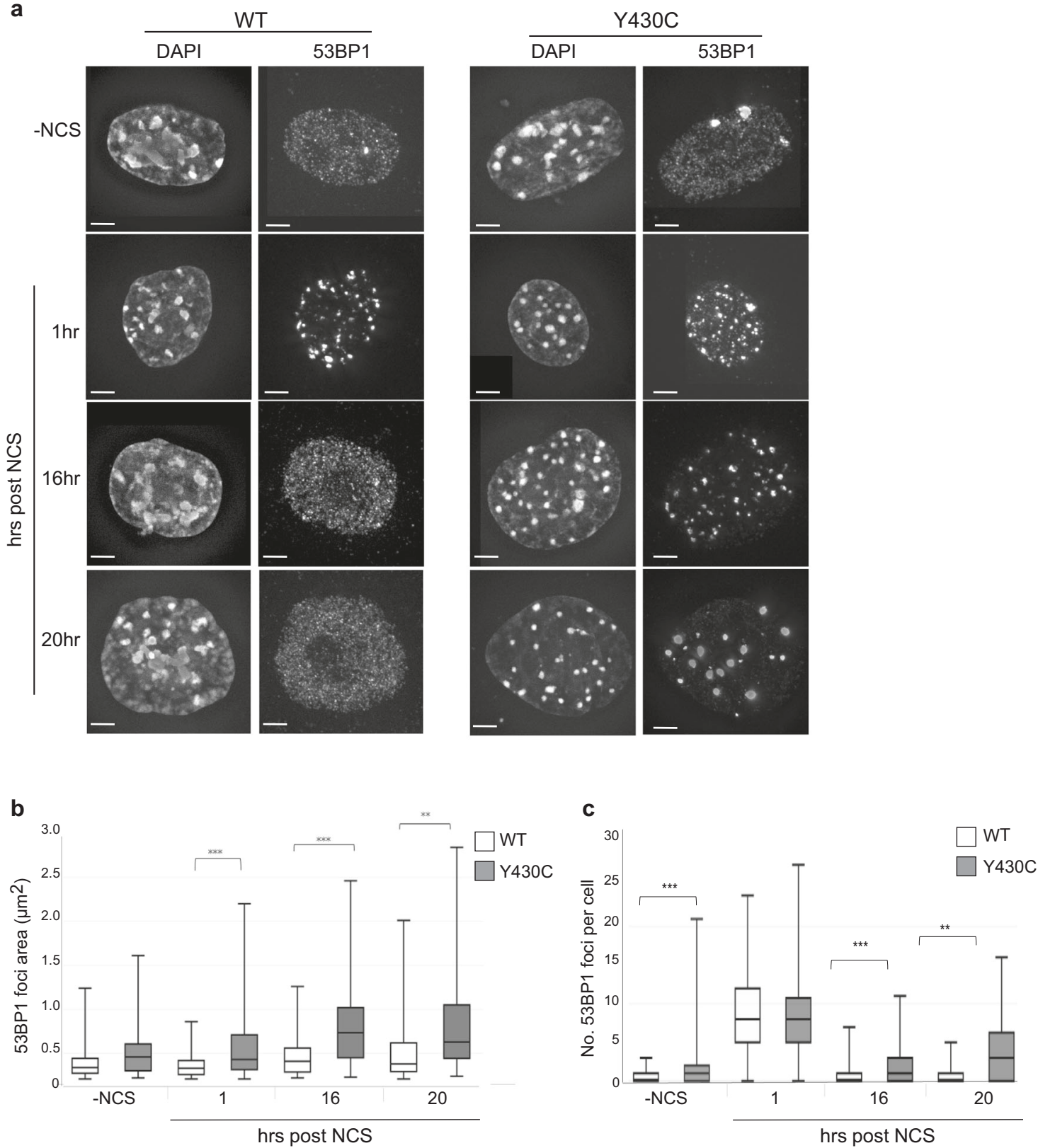

Fig. 4 Increased size and number of 53BP1 foci after DSB induction in Y430C mESCs. a Immunofluorescence for 53BP1 in the DAPI-stained nuclei of wild-type and Y430C mESCs upon treatment with NCS and after recovery periods up to $20 \mathrm{~h}$. Scale bars: $2 \mu \mathrm{m}$. The experiment was repeated 3 times independently with similar results. b, c Box-plots show area $\left(\mu \mathrm{m}^{2}\right)$ and number of 53BP1 foci per cell, respectively, in WT and Y430C cells after treatment with NCS in one representative experiment. Horizontal lines within boxes show medians, boxes are inter-quartile ranges and whiskers are range. $P$-values were calculated with Mann-Whitney one-sided $U$ test. ${ }^{\star}<0.05,{ }^{\star \star}<0.01,{ }^{\star \star \star}<0.001 .50$ cells per condition were examined. Data from three independent experiment are in Supplementary Fig. 6.

end-resection, we propose that the Y430C BRD4 mutation leads to an altered balance between NHEJ and HR, consistent with the synthetic lethality observed between BRD4 and PARP inhibitors 25,28 .

Increased number and size of 53BP1 foci in NIPBL mutant lymphoblastoid cell lines. To see if the DDR defect that we have observed in the presence of the $\mathrm{BRD} 4^{\mathrm{Y} 430 \mathrm{C}}$ would also be apparent in cells carrying other CdLS mutations, we utilised two lymphoblastoid cell lines (LCL) previously derived from CdLS patients with heterozygous mutations in NIPBL, Ile1206del ${ }^{36}$ and Arg2298 $\mathrm{His}^{37}$. These LCLs have significantly more, and larger, 53BP1 foci per cell compared to a WT LCL, in the absence of any exogenous damage (Fig. 6c-e, Supplementary Fig. 8a, b). This suggests that increased DDR signalling and/or impaired DNA repair pathway choice balance may be a common mechanism underlying CdLS caused by BRD4 and NIPBL.

\section{Discussion}

We previously showed that a Y430C-BRD4 mutation, and BRD4 haploinsufficiency, cause a CdLS-like syndrome ${ }^{8}$. The severe developmental phenotypes associated with CdLS are generally thought to be due to aberrant gene regulation. Here, however, we show that BRD4 ${ }^{\mathrm{Y} 430 \mathrm{C}}$, whilst lowering the affinity of BRD4 to acetylated lysine residues and decreasing its occupancy at enhancers and SEs, causes minor changes in transcription in 
a
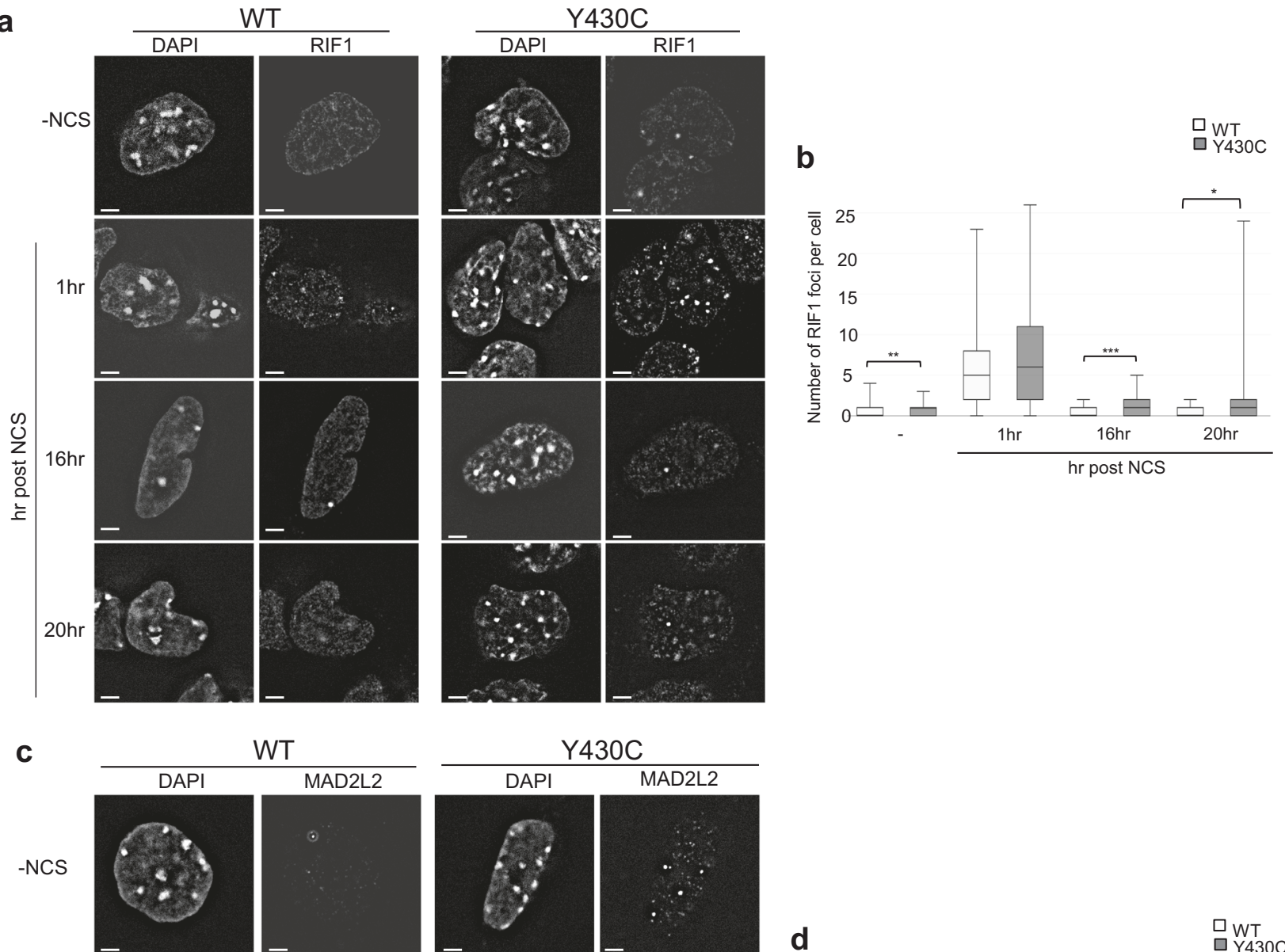

WT
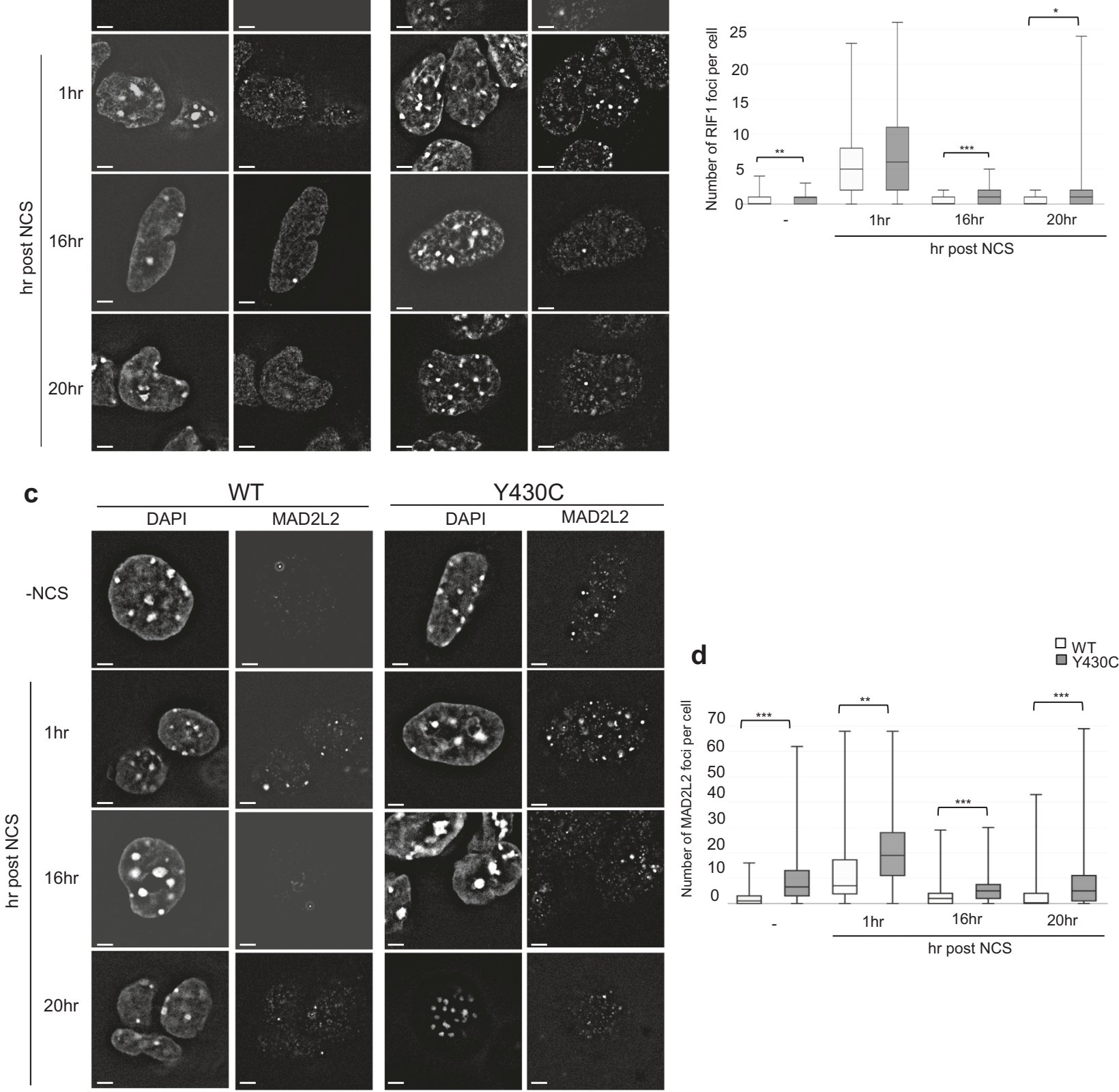

Fig. 5 Increased RIF1 and MAD2L2 foci after DSB induction in Y430C mESCs. a Representative images of wild-type and Y430C mESCs upon RIF1 immunofluorescence and DAPI staining after treatment with NCS. Scale bars: $2 \mu \mathrm{m}$. The experiment was repeated three times with similar results. $\mathbf{b}$ Boxplot shows number of RIF1 foci per cell, respectively, in WT and Y430C cells after treatment with NCS in one representative experiment. Horizontal lines within boxes show medians, boxes are inter-quartile ranges and whiskers are range. P-values were calculated with Mann-Whitney one-sided $U$ test. ${ }^{\star}<0.05,{ }^{\star \star}<0.01,{ }^{\star \star \star}<0.001 .50$ cells were examined per condition. c Representative images of wild-type and Y430C mESCs upon MAD2L2 immunofluorescence and DAPI staining after treatment with NCS. Scale bars: $2 \mu \mathrm{m}$. The experiment was repeated three times with similar results. $\mathbf{d}$ Boxplot shows number of MAD2L2 foci per cell, respectively, in WT and Y $430 \mathrm{C}$ cells after treatment with NCS in one representative experiment. Horizontal lines within boxes show medians, boxes are inter-quartile ranges and whiskers are range. $P$-values were calculated with Mann-Whitney one-sided $U$ test. ${ }^{*}<0.05,{ }^{* *}<0.01,{ }^{* * *}<0.001 .50$ cells were examined per condition.

mESCs, in contrast to the major transcriptional changes caused by the profound loss of BRD4 binding induced by BET inhibitors. Instead, we provide evidence that the $\mathrm{BRD} 44^{\mathrm{Y} 430 \mathrm{C}}$ hypomorph causes increased G2/M checkpoint activation, aberrant DDR signalling and an altered focal accumulation of proteins that promote NHEJ and inhibit HR-53BP1 and the shieldin complex. Conversely there is a depletion of foci containing HR proteins (Rad51), suggesting a defect in HR. Our results suggest a role for BRD4 in the regulation of DNA repair pathway choice and are consistent with recent results from an siRNA screen in human 

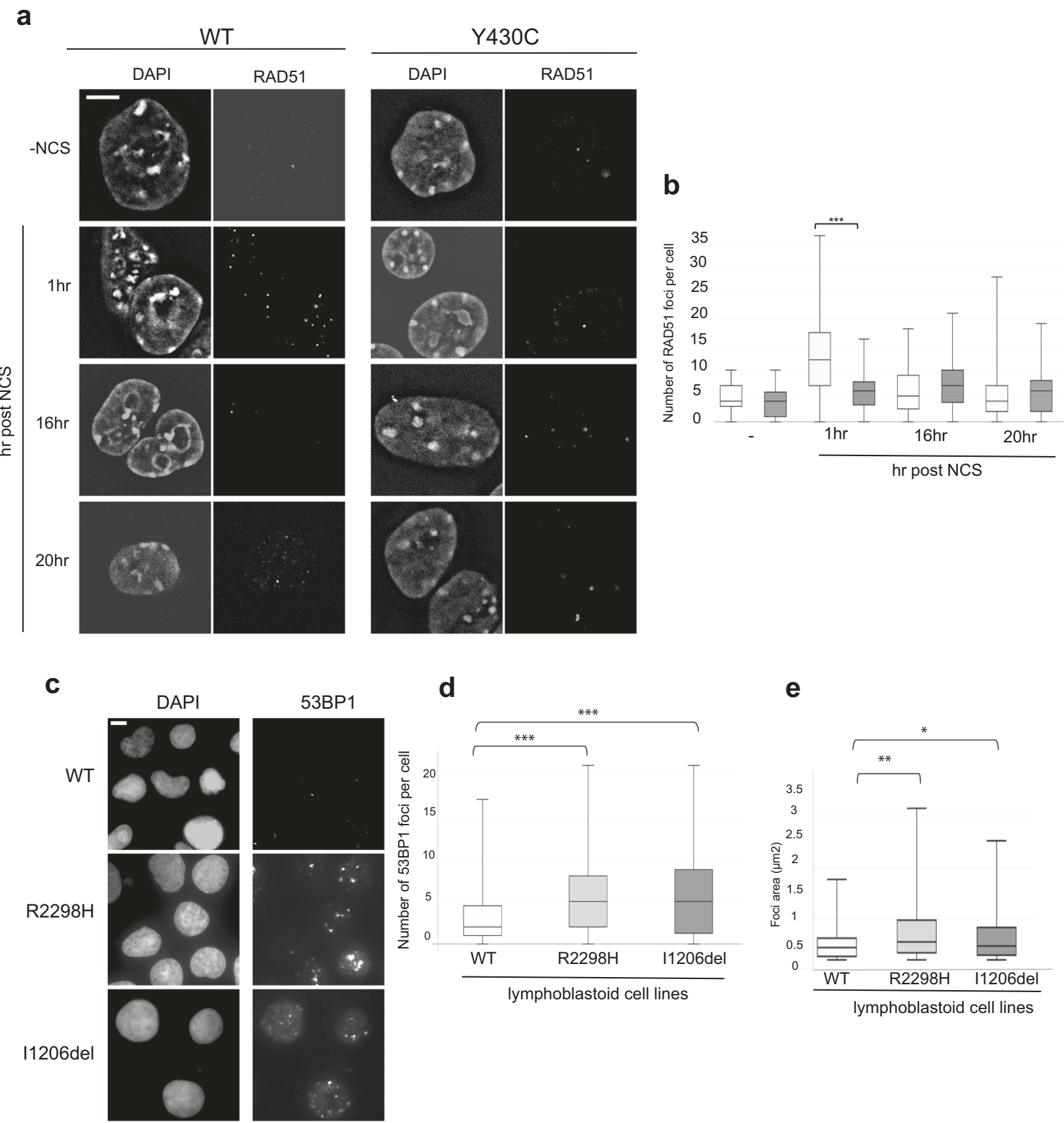

Fig. 6 Evidence for DNA repair defects in CdLS. Representative images of wild-type and Y430C mESCs upon RAD51 immunofluorescence and DAPI staining after treatment with NCS. Scale bar $=5 \mu \mathrm{m}$. The experiment was repeated three times with similar results. b Box-plot shows number of RAD51 foci per cell, respectively, in WT and Y430C cells after treatment with NCS in one representative experiment. Horizontal lines within boxes show medians, boxes are inter-quartile ranges and whiskers are range. $P$-values were calculated with Mann-Whitney one-sided $U$ test. ${ }^{\star}<0.05,{ }^{* \star}<0.01,{ }^{\star \star \star}<0.001 .50$ cells were examined per condition. c Representative images of wild-type, R2298 and I1206del LCLs upon 53BP1 and DAPI immunofluorescence. Scale bar $=5 \mu \mathrm{m}$. The experiment was repeated three times with similar results. d, e Box-plots show number of 53BP1 foci per cell and area of 53BP1 foci ( $\mu \mathrm{m}^{2}$ ), respectively, in WT, R2298H and 11206 del LCLs in one representative experiment. Horizontal lines within boxes show medians, boxes are inter-quartile ranges and whiskers are range. $P$-values were calculated with Mann-Whitney one-sided $U$ test. ${ }^{\star}<0.05,{ }^{* \star}<0.01,{ }^{* \star *}<0.001$. 50 cells were examined per condition.

cell lines that suggest that BRD4 deficiency leads to less repair by $\mathrm{HR}$ and more by $\mathrm{NHEJ}^{38}$. Whether BRD4 mutation affects repair by $\mathrm{HR}$ at specific regions in the genome, or globally, remains to be investigated. For example, different levels of histone acetylation in different chromatin environments-e.g. heterochromatin vs euchromatin-upon DNA damage may recruit different amounts of BRD4 $4^{39,40}$. Alternatively, as well as interacting with NIPBL, Brd4 has been reported to interact with a number of proteins involved in DNA damage sensing and repair, including Rif1 of the Sheildin complex. . $^{8,4}$ We cannot exclude that impaired $\mathrm{BRD} 4$ function in $\mathrm{BRD} 4{ }^{\mathrm{Y} 430 \mathrm{C}}$ cells affects the formation of DSBs themselves, for example by preventing the accumulation of R-loops ${ }^{23,38,42}$.

We observed a similar focal accumulation of 53BP1 in cells from CdLS patients with mutations in NIPBL. Could aberrant DDR and DNA repair choice, or perhaps elevated DNA damage 
per se, therefore account for some of the phenotypes associated with CdLS? Congenital mutation in many different genes involved in cell cycle progression and DNA repair, are-like CdLS-generally associated with intrauterine growth retardation and short stature ${ }^{43}$. Similarly, microcephaly also results from mutation in genes associated with $S$ phase progression (ATR, ATRIP, CtIP-Seckel syndrome; DNA ligase IV-lig4 syndrome; XRCC4-microcephalic primordial dwarfism ${ }^{44-46}$ ). Clinically, there is strongest phenotypic overlap between CdLS and Rubinstein-Taybi syndromes (RTS) - including arched eyebrows and other shared distinctive facial features. RTS is caused by mutations in p300 or CREBBP. These lysine acetyltransferases have recently been shown to be important for acetylating proteins involved in the DDR and DNA repair ${ }^{47}$. NIPBL and cohesin are also both involved in DNA damage signalling and repair ${ }^{48}$ and CdLS patient cells carrying NIPBL mutations display an increased DNA damage sensitivity ${ }^{49}$. Even though we cannot discount that BRD4 mutation in CdLS cases-Y430C, or heterozygous deletions, cause aberrant transcriptional regulation in cell types other than ESCs, our results suggest that dysregulation of DDR and repair may contribute to the aetiology of CdLS.

\section{Methods}

Cell culture. Y430C-BRD4 mutant and corresponding wild-type mouse embryonic stem cells (mESCs) were generated by CRISPR Cas9 genome editing in $46 \mathrm{C}$ mESCs as described previously ${ }^{8}$. NIPBL I1206del and R2298H lymphoblastoid cell lines (LCLs) were obtained from patients ${ }^{36,37}$. mESCs were cultured in GMEM medium (GIBCO; 11710035) supplemented with 10\% Fetal Calf Serum (FCS), 5\% penicillin-streptomycin, $1 \mathrm{mM}$ sodium pyruvate (GIBCO; 11360070), 1X nonessential amino acids (GIBCO; 11140050), $50 \mu \mathrm{M}$ 2-mercaptoethanol (GIBCO 31350010), $2 \mathrm{mM} \mathrm{L}$-glutamine and 500U/ml Leukaemia Inhibitory Factor (in house). Lymphoblastoid cell lines (LCLs) were grown in RPMI 1640 medium (GIBCO; 11875093) supplemented with 15\% FCS and $2 \mathrm{mM} \mathrm{L-glutamine.} \mathrm{All} \mathrm{cells}$ were grown at $37^{\circ} \mathrm{C}$ in a $5 \% \mathrm{CO}_{2}$ humidified atmosphere.

ChIP-qPCR. Cells were harvested by trypsinising and fixed with $1 \%$ formaldehyde (Thermo Fisher; 28906$)$ in media $\left(25^{\circ} \mathrm{C}, 10 \mathrm{~min}\right)$. This reaction was quenched with $0.125 \mathrm{M}$ glycine for $5 \mathrm{~min}$. ChIP-qPCR was performed as described previously ${ }^{8}$ (see Table 1 for antibodies). DNA was purified using the QIAquick PCR Purification kit (Qiagen, 28104). Input samples were diluted to $1 \%$, and all samples diluted a further 10 -fold, in $\mathrm{ddH}_{2} 0$. SYBR-green based qPCR reactions were performed in a final volume of $20 \mu \mathrm{l}$ containing diluted ChIP DNA, SYBR select master mix (ThermoFisher Scientific; 4472908) and $0.25 \mu \mathrm{M} / \mathrm{L}$ of each primer (see Table 1). Concentration of IPs are relative to $1 \%$ input.

ChIP-seq. ChIP was carried out as above. After purification, DNA was eluted in $20 \mu \mathrm{l}$ and libraries were prepared for ChIP and input samples as previously described ${ }^{50}$. Samples were sequenced at BGI (Hong Kong; 50-bp single-end reads) using the HiSeq 4000 system (Illumina). Fastq files were quality controlled using FastQC and mapped to the $\mathrm{mm} 9$ genome using Bowtie2 (parameters: default). Sam files were converted to bam files and sorted using SamTools. Homer was used to make tagdirectories (makeTagDirectory, parameters: -unique, fragLength 150) and bedgraphs (makeUCSCfile, parameters: default). For visualisation of BRD4 data, bedgraphs were uploaded to the genome browser UCSC. Peak calling was carried out using MACS2; duplicates were filtered (filterdup, parameters:-keep-dup=1), peaks called (callpeaks, parameters: -B-nomodel -p 1e-5) and differential peaks were found (bdgdiff, parameters: -g $60-1250$ ).

deepTools2 was used to make heatmaps; score files were made across specific genomic regions (computeMatrix, parameters: scale-regions scale regions -b 500 -a 500 -bs $50-\mathrm{bl} \mathrm{mm} 9$ blacklist) and these were used to plot heatmaps (plotHeatmap, parameters:-colormap RdBluYl reverse).

JQ1 treatment. $1 \mathrm{mM}$ BRD4 inhibitor JQ1 +, or its inactive form JQ1- (Merck; 500586) (diluted in DMSO), were added to $\mathrm{mESC}$ media at a final concentration of $300 \mathrm{nM}$. JQ1 $1 /$ - . WT and Y430C mESCs were incubated at $37^{\circ} \mathrm{C}$ with JQ1 $+/$ supplemented media for $48 \mathrm{~h}$. Total RNA was extracted from cells using the RNeasy Plus Mini Kit (Qiagen; 74134) and $1 \mu \mathrm{g}$ RNA was used for cDNA synthesis with SuperScript II Reverse Transcriptase (ThermoFisher Scientific; 18064-014) as per manufacturer's instructions. cDNA was diluted 1:500 for qPCR analysis. qPCR reactions were performed as above (see Table 1 for primers). Concentration of JQ1 + cDNA was calculated relative to JQ1- (arbitrarily set to 1 ).
RT-PCR. RNA was extracted from cells using the RNeasy Mini Kit (Qiagen; 74104) using spin technology, with an additional on-column DNA digestion using the RNase-Free DNase Set (Qiagen; 79254). cDNA was synthesised from $1 \mu \mathrm{g}$ RNA using SuperScript II Reverse Transcriptase (ThermoFisher Scientific; 18064-014) as per manufacturer's instructions. cDNA was diluted 1 in 25 for qPCR analysis. SYBR-green based qPCR reactions were performed in a final volume of $20 \mu \mathrm{l}$ containing diluted cDNA, SYBR select master mix (ThermoFisher Scientific; $4472908)$ and $0.5 \mu \mathrm{M} / \mathrm{l}$ of region specific intron-spanning primer pairs.

4sU-seq. 4sU RNA was generated and isolated as described previously ${ }^{51}$, with the following changes: cells were incubated at $37^{\circ} \mathrm{C}$ with $4 \mathrm{sU}$-supplemented medium for $20 \mathrm{~min}$. The reaction was incubated with Biotin-HPDP with rotation for $1.5 \mathrm{~h}$ at RT. For recovery of biotinylated $4 \mathrm{sU}-\mathrm{RNA}, 1 \mu \mathrm{l}$ of streptavidin beads was added per $\mu \mathrm{g}$ of RNA. Columns were washed using $900 \mu \mathrm{l}$ washing buffer and RNA was eluted by 2 sequential additions of $100 \mu$ l Elution Buffer (100 mM DTT) to the column and eluates combined. RNA was further purified using the RNAeasy MinElute Clean-up kit (Qiagen; 74204) according to the manufacturer's guidelines, eluting in $20 \mu \mathrm{l}$ water. $1 \mu \mathrm{l}$ of $4 \mathrm{~s} U$-labelled RNA was quality-checked by running on a 2100 Bioanalyzer Instrument (Agilent).

To make $4 \mathrm{sU}$ sequencing libraries, $4 \mathrm{~s} \mathrm{U}$ labelled RNA was first depleted of rRNA using the Low Input Ribominus Eukaryotic System V2 (ThermoFisher Scientific; A15027) as per the manufacturer's instructions. $600 \mathrm{ng}$ of $4 \mathrm{sU}$ labelled RNA was used as input, and eluted in $5 \mu \mathrm{l}$ RNase free water. All of the resulting rRNA free RNA was used to prepare $4 \mathrm{sU}$ sequencing libraries, using NEBnext Ultra Directional RNA library prep kit of Illumina (NEB; E7420). RNA fragmentation was carried out at $94^{\circ} \mathrm{C}$ for $15 \mathrm{~min}$, as suggested for intact RNA. Libraries were indexed with Multiplex Oligos for Illumina (Index Primers Set 1) (NEBnext; E7335) and amplified by PCR for 13 cycles. Library concentration and correct size distribution was confirmed on the Agilent 2100 Bioanalyser with the DNA HS Kit. Libraries were sequenced at BGI (Hong Kong; 100-base paired-end reads) using the HiSeq 4000 system (Illumina).

Fastq files were quality controlled using FastQC and mapped to the $\mathrm{mm} 9$ genome using tophat (parameters:-library-type fr-firststrand -r 200). Homer was used to make tagdirectories (makeTagDirectory, parameters: -unique -sspe -flip -fragLength 150), and to make bedgraphs for visualisation on UCSC (makeUCSCfile, parameters: -strand separate -style rnaseq). Cufflinks was used for peak calling; transcripts were assembled for individual experiments (cufflinks, parameters: -m 200 -library-type fr) and both replicates of WT and Y430C were combined to form one assembly (cuffmerge, parameters: default). Differentially expressed peaks were determined from this assembly using cuffdiff (Cuffdiff. Parameters: default).

Heatmaps were generated as above.

Spike-in RNA-seq. S2 cells were cultured in Schneider's Drosophila Medium (Invitrogen; 11720-034), supplemented with 10\% heat-inactivated FCS and 5\% penicillin-streptomycin. Cells were passaged once they reached a density of $\sim 2 \times$ $10^{7} \mathrm{cells} / \mathrm{ml}$ and seeded at a density of $\sim 4 \times 10^{6}$. Cells were grown at $28^{\circ} \mathrm{C}$ in a $5 \%$ $\mathrm{CO}_{2}$ humidified atmosphere. Cells were frozen at a density of $\sim 1 \times 10^{7} \mathrm{cells} / \mathrm{ml}$ in $45 \%$ conditioned Schneider's Drosophila Medium media (containing 10\% FCS), 45\% fresh Schneider's Drosophila Medium supplemented with 10\% FCS, and 10\% DMSO, and stored in liquid nitrogen.

mESCs and S2 cells were harvested and counted. 0.2 million S2 cells were mixed with 10 million mESCs, and RNA was extracted using the RNeasy Mini Kit (Qiagen; 74104) using spin technology, with an additional on-column DNA digestion using the RNase-Free DNase Set (Qiagen; 79254). RNA was depleted of rRNA and RNA-seq libraries prepared as for the 4 sU-seq.

Growth assay. WT and Y430C mESCs were each seeded in 4 wells of a 6 well plate $\left(1 \times 10^{4}\right.$ cells/well). WT and Y430C cells from 1 well were trypsinised and counted at $24,48,72$ and $96 \mathrm{~h}$ post seeding. Counting was carried out manually using a haemocytometer. The addition of trypan blue dye allowed for the exclusion of dead cells.

Flow cytometry. 2 million mESCs were fixed in $70 \%$ ethanol (in PBS) at $4^{\circ} \mathrm{C}$ for $1 \mathrm{~h}$. Fixed cells were centrifuged at $300 \mathrm{~g}$ at $4^{\circ} \mathrm{C}$ for $5 \mathrm{~min}$, washed twice with PBS and resuspended in $500 \mu \mathrm{l}$ PBS. $20 \mu \mathrm{g}$ RNase A was added and cells were incubated at $37^{\circ} \mathrm{C}$ for $10 \mathrm{~min}$. Cells were stained with propium iodide at a final concentration of $50 \mu \mathrm{g} / \mathrm{ml}$. Acquisition was carried out on a BD LSRFortessa cell analyser, collecting 25,000 events per sample. Results were analysed using BD FACSDiva 8.0.1 and gated cells were manually categorised into cell cycle stages G0/G1, S and G2/M.

NCS treatment and CHK-1 protein Western blots. Cells were incubated with mESC media supplemented with neocarzinostatin (Sigma; N9162) (NCS), to a fina concentration of $25 \mathrm{ng} / \mathrm{ml}$, for $15 \mathrm{~min}$ at $37^{\circ} \mathrm{C}$. Cells were then washed with PBS and fresh, non-supplemented media was added. Protein was either extracted straight away, or after incubation at $37^{\circ} \mathrm{C}$ for varying lengths of time. Ice-cold RIPA buffer (150 mM sodium chloride; $1.0 \%$ NP-40; $0.5 \%$ sodium deoxycholate; $0.1 \%$ SDS; $50 \mathrm{mM}$ Tris, $\mathrm{pH} 8.0)$ was added to plates $\left(1 \mathrm{ml}\right.$ per $10^{7}$ cells $)$ and cells 
Table 1 Key resources.

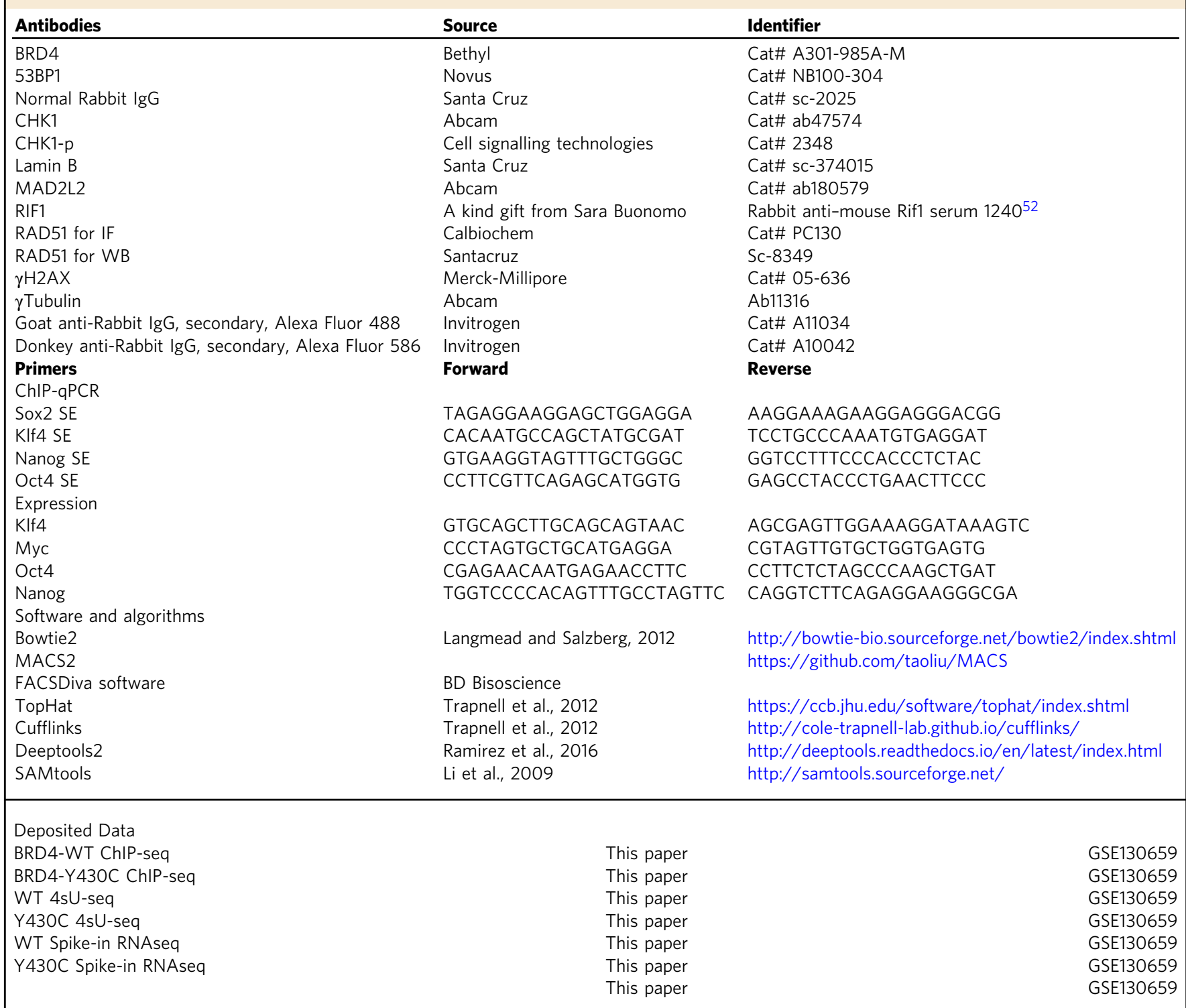

were scraped and transferred into pre-chilled microcentrifuge tubes. Tubes were shaken at $4{ }^{\circ} \mathrm{C}$ for $30 \mathrm{~min}$ before centrifugation at $20,000 \mathrm{xg}$ for $15 \mathrm{~min}$. Supernatant was retained and quantified. For Western blot analysis, equal amounts of protein were boiled in 1X NuPage LDS buffer (ThermoFisher Scientific, NP0008) with 1X NuPage reducing agent (ThermoFisher Scientific; NP0004) for 5 min and separated on a 3-8\% tris-acetate gel (ThermoFisher Scientific; EA0375BOX). Following electrophoresis, proteins were transferred to nitrocellulose membranes (ThermoFisher Scientific) and immunoblotted with primary antibodies overnight at $4{ }^{\circ} \mathrm{C}$. Membranes were washed $3 \times$ TBST and probed with HRP-conjugated secondary antibody for $1 \mathrm{~h}$ at RT. After 3 more washes in TBST, membranes were incubated with SuperSignal ${ }^{\text {me }}$ West Femto Maximum Sensitivity Substrate (ThermoFisher Scientific; 34095) for 5 min and imaged using ImageQuant ${ }^{\text {tm }}$ LAS 4000 (GE Healthcare).

Immunofluorescence. mESCs for immunofluorescence experiments were cultured on gelatinised coverslips and LCLs were grown in suspension. LCLs were harvested and resuspended in PBS to $1.8 \times 10^{5} \mathrm{cells} / \mathrm{ml}$. $500 \mu \mathrm{l}$ of cell suspension was added to a Shandon ${ }^{\text {tw }}$ Single Cytofunnel ${ }^{\text {mot }}$ (ThermoFisher Scientific; 5991040), with a microscope slide attached. Slides were centrifuged at $800 \mathrm{rpm}$ for $5 \mathrm{~min}$, after which the LCLs had attached to the slide. All cells were fixed in $4 \%$ paraf ormaldehyde for $10 \mathrm{~min}$ and washed $3 \times 3 \mathrm{~min}$ in PBS. Cells were then permeabilised in $0.5 \%$ Triton in PBS for $10 \mathrm{~min}$ and washed $3 \times 3 \mathrm{~min}$ in PBS. Cells were blocked in $1 \%$ BSA in PBS for $30 \mathrm{~min}$ at RT, incubated with primary antibody diluted in $1 \%$ BSA for $1 \mathrm{~h}$ at RT and washed $3 \times 3 \mathrm{~min}$ in PBS. Cells were next incubated with secondary antibody (see Table 1) diluted in $1 \%$ BSA for 45 min at
RT, washed $3 \times 3 \mathrm{~min}$ in PBS, incubated with DAPI in PBS $(250 \mathrm{ng} / \mathrm{ml})$ for $2 \mathrm{~min}$, and washed $3 \times 3 \mathrm{~min}$ in PBS. Coverslips were mounted on slides in Vectashield (Vector; H1000) mounting medium for fluorescence.

All slides were viewed, and foci counted, using epifluorescence microscopes. Images were taken using confocal microscopy.

Laser microirradiation of U20S cells. U2OS cells $\left(\right.$ ATCC $^{\circ}$ HTB- $\left.96^{\mathrm{mm}}\right)$ were grown on glass coverslips and incubated with $10 \mu \mathrm{M}$ BrdU (Sigma Aldrich, \#B9285) for $24 \mathrm{~h}$ prior to microirradiation. Microirradiation was induced with a $405 \mathrm{~nm}$ laser diode $(3 \mathrm{~mW})$ focused through a $63 \mathrm{x} / 1.4$ oil objective on a Zeiss LSM710 confocal microscope using the following laser settings: $40 \%$ power, 50 iterations, scan speed 12.6 $\mu \mathrm{sec} /$ pixel. Cells were fixed either $10 \mathrm{~min}$ or $2 \mathrm{~h}$ after laser irradiation using $2 \%$ PFA and immunofluorescence was performed as described in the immunofluorescence section using $\gamma \mathrm{H} 2 \mathrm{AX}$ antibody (Merck-Millipore, \#05-636) as positive control of DNA damage induction. Image acquisition was performed on a Leica DMI6000 epifluorescence microscope using a Plan-Apochromat 40x/1.3 oil objective.

Reporting summary. Further information on research design is available in the Nature Research Reporting Summary linked to this article.

\section{Data availability}

Data supporting the findings of this study have been deposited in GEO with accession number GSE130659. All data is available from the authors upon reasonable request. Source data are provided with this paper. 


\section{Code availability statement}

Custom script for analysis of foci area is deposited here: https:/github.com/IGMMImagingFacility/Boumendil2020_BRD4paper; https://doi.org/10.5281/zenodo.4596974.

Received: 10 June 2019; Accepted: 29 April 2021;

Published online: 25 May 2021

\section{References}

1. Kline, A. D. et al. Diagnosis and management of Cornelia de Lange syndrome: first international consensus statement. Nat. Rev. Genet. 19, 649 (2018).

2. Ansari, M. et al. Genetic heterogeneity in Cornelia de Lange syndrome (CdLS) and CdLS-like phenotypes with observed and predicted levels of mosaicism. J. Med. Genet. 51, 659-668 (2014).

3. Castronovo, P. et al. Premature chromatid separation is not a useful diagnostic marker for Cornelia de Lange syndrome. Chromosome Res. 17, 763-771 (2009).

4. Mehta, D. et al. Characterization of limb differences in children with Cornelia de Lange Syndrome. Am. J. Med. Genet. Part C 172, 155-162 (2016).

5. Remeseiro, S., Cuadrado, A. \& Losada, A. Cohesin in development and disease. Development 140, 3715-3718 (2013).

6. Dorsett, D. The Drosophila melanogaster model for Cornelia de Lange syndrome: implications for etiology and therapeutics. Am. J. Med. Genet. C. Semin. Med. Genet. 172, 129-137 (2016).

7. Vrouwe, M. G. et al. Increased DNA damage sensitivity of Cornelia de Lange syndrome cells: evidence for impaired recombinational repair. Hum. Mol. Genet. 16, 1478-1487 (2007).

8. Olley, G. et al. BRD4 interacts with NIPBL and BRD4 is mutated in a Cornelia de Lange-like syndrome. Nat. Genet. https://doi.org/10.1038/s41588-0180042-y (2018).

9. Di Micco, R. et al. Control of embryonic stem cell identity by brd4-dependent transcriptional elongation of super-enhancer-associated pluripotency genes. Cell Rep. 9, 234-247 (2014).

10. Lovén, J. et al. Selective inhibition of tumor oncogenes by disruption of superenhancers. Cell 153, 320-334 (2013)

11. Rahman, S. et al. The Brd4 extraterminal domain confers transcription activation independent of pTEFb by recruiting multiple proteins, including NSD3. Mol. Cell. Biol. 31, 2641-2652 (2011).

12. Jang, M. K. et al. The bromodomain protein Brd4 is a positive regulatory component of $\mathrm{P}-\mathrm{TEFb}$ and stimulates RNA polymerase II-dependent transcription. Mol. Cell 19, 523-534 (2005).

13. Dev, H. et al. Shieldin complex promotes DNA end-joining and counters homologous recombination in BRCA1-null cells. Nat. Cell Biol. 20, 954-965 (2018).

14. Ghezraoui, H. et al. 53BP1 cooperation with the REV7-shieldin complex underpins DNA structure-specific NHEJ. Nature 560, 122-127 (2018).

15. Gupta, R. et al. DNA repair network analysis reveals shieldin as a key regulator of NHEJ and PARP inhibitor sensitivity. Cell 173, 972-988.e23 (2018).

16. Mirman, Z. et al. 53BP1-RIF1-shieldin counteracts DSB resection through CST- and Pola-dependent fill-in. Nature 560, 112-116 (2018).

17. Noordermeer, S. M. et al. The shieldin complex mediates 53BP1-dependent DNA repair. Nature 560, 117-121 (2018).

18. Boersma, V. et al. MAD2L2 controls DNA repair at telomeres and DNA breaks by inhibiting 5 ' end resection. Nature 521, 537-540 (2015).

19. Chapman, J. R. et al. RIF1 is essential for 53BP1-dependent nonhomologous end joining and suppression of DNA double-strand break resection. Mol. Cell 49, 858-871 (2013).

20. Feng, L., Fong, K.-W., Wang, J., Wang, W. \& Chen, J. RIF1 counteracts BRCA1-mediated end resection during DNA repair. J. Biol. Chem. 288, 11135-11143 (2013).

21. Di Virgilio, M. et al. Rif1 prevents resection of DNA breaks and promotes immunoglobulin class switching. Science 339, 711-715 (2013).

22. Escribano-Díaz, C. et al. A cell cycle-dependent regulatory circuit composed of 53BP1-RIF1 and BRCA1-CtIP controls DNA repair pathway choice. Mol. Cell 49, 872-883 (2013)

23. Floyd, S. R. et al. The bromodomain protein Brd4 insulates chromatin from DNA damage signalling. Nature 498, 246-250 (2013).

24. Li, X. et al. BRD4 promotes DNA repair and mediates the formation of TMPRSS2-ERG gene rearrangements in prostate cancer. Cell Rep. 22, 796-808 (2018).

25. Wilson, A. J., Stubbs, M., Liu, P., Ruggeri, B. \& Khabele, D. The BET inhibitor INCB054329 reduces homologous recombination efficiency and augments PARP inhibitor activity in ovarian cancer. Gynecologic Oncol. 149, 575-584 (2018).

26. Pongas, G. et al. BRD4 facilitates DNA damage response and represses CBX5/ Heterochromatin protein 1 (HP1). Oncotarget 8, 51402-51415 (2017).
27. Kastan, M. B. \& Bartek, J. Cell-cycle checkpoints and cancer. Nature 432, 316 (2004).

28. Sun, C. et al. BRD4 inhibition is synthetic lethal with PARP inhibitors through the induction of homologous recombination deficiency. Cancer Cell 33, 401-416.e8 (2018).

29. Gong, F. et al. Screen identifies bromodomain protein ZMYND8 in chromatin recognition of transcription-associated DNA damage that promotes homologous recombination. Genes Dev. 29, 197-211 (2015).

30. Turinetto, V. et al. High basal $\gamma \mathrm{H} 2 \mathrm{AX}$ levels sustain self-renewal of mouse embryonic and induced pluripotent stem cells. Stem Cells 30, 1414-1423 (2012).

31. Panier, S. \& Boulton, S. J. Double-strand break repair: 53BP1 comes into focus. Nat. Rev. Mol. Cell Biol. 15, 7-18 (2014).

32. Harrigan, J. A. et al. Replication stress induces 53BP1-containing OPT domains in G1 cells. J. Cell Biol. 193, 97-108 (2011).

33. Lukas, C. et al. 53BP1 nuclear bodies form around DNA lesions generated by mitotic transmission of chromosomes under replication stress. Nat. Cell Biol. 13, 243-253 (2011)

34. Bowry, A., Piberger, A. L., Rojas, P., Saponaro, M. \& Petermann, E. BET inhibition induces HEXIM1- and RAD51-dependent conflicts between transcription and replication. Cell Rep. 25, 2061-2069.e4 (2018).

35. Shrivastav, M., De Haro, L. P. \& Nickoloff, J. A. Regulation of DNA doublestrand break repair pathway choice. Cell Res. 18, 134-147 (2008).

36. Tonkin, E. T., Wang, T.-J., Lisgo, S., Bamshad, M. J. \& Strachan, T. NIPBL, encoding a homolog of fungal Scc2-type sister chromatid cohesion proteins and fly Nipped-B, is mutated in Cornelia de Lange syndrome. Nat. Genet. 36, 636-641 (2004).

37. Gillis, L. A. et al. NIPBL mutational analysis in 120 individuals with Cornelia de Lange syndrome and evaluation of genotype-phenotype correlations. Am. J. Hum. Genet. 75, 610-623 (2004).

38. Kim, J. J. et al. Systematic bromodomain protein screens identify homologous recombination and R-loop suppression pathways involved in genome integrity. Genes Dev. 33, 1751-1774 (2019).

39. Clouaire, T. \& Legube, G. DNA double strand break repair pathway choice: a chromatin based decision. Nucleus 6, 107-113 (2015).

40. Sun, Y. et al. Histone H3 methylation links DNA damage detection to activation of the tumour suppressor Tip60. Nat. Cell Biol. 11, 1376-1382 (2009).

41. Lambert, J.-P. et al. Interactome rewiring following pharmacological targeting of BET bromodomains. Mol. Cell 73, 621-638.e17 (2019).

42. Lam, F. C. et al. BRD4 prevents the accumulation of R-loops and protects against transcription-replication collision events and DNA damage. Nat. Commun. 11, 4083 (2020).

43. Logan, C. V. et al. DNA polymerase epsilon deficiency causes IMAGe syndrome with variable immunodeficiency. Am. J. Hum. Genet. 103, 1038-1044 (2018).

44. Rosin, N. et al. Mutations in XRCC4 cause primary microcephaly, short stature and increased genomic instability. Hum. Mol. Genet. 24, 3708-3717 (2015).

45. Murray, J. E. et al. Mutations in the NHEJ component XRCC4 cause primordial dwarfism. Am. J. Hum. Genet. 96, 412-424 (2015).

46. O’Driscoll, M., Gennery, A. R., Seidel, J., Concannon, P. \& Jeggo, P. A. An overview of three new disorders associated with genetic instability: LIG4 syndrome, RS-SCID and ATR-Seckel syndrome. DNA Repair 3, 1227-1235 (2004)

47. Dutto, I., Scalera, C. \& Prosperi, E. CREBBP and p300 lysine acetyl transferases in the DNA damage response. Cell. Mol. Life Sci. 75, 1325-1338 (2018).

48. Litwin, I., Pilarczyk, E. \& Wysocki, R. The emerging role of cohesin in the DNA damage response. Genes $\mathbf{9}, 581$ (2018)

49. Pastink, A. et al. Increased DNA damage sensitivity of Cornelia de Lange syndrome cells: evidence for impaired recombinational repair. Hum. Mol. Genet. 16, 1478-1487 (2007).

50. Pradeepa, M. M. et al. Histone H3 globular domain acetylation identifies a new class of enhancers. Nat. Genet. 48, 681-686 (2016).

51. Pintacuda, G. et al. hnRNPK recruits PCGF3/5-PRC1 to the Xist RNA Brepeat to establish polycomb-mediated chromosomal silencing. Mol. Cell 68 , 955-969.e10 (2017).

52. Buonomo, S. B. C., Wu, Y., Ferguson, D. \& De Lange, T. Mammalian Rif 1 contributes to replication stress survival and homology-directed repair. J. Cell Biol. https://doi.org/10.1083/jcb.200902039 (2009).

\section{Acknowledgements}

We thank the individuals with CdLS and their families for generously donating their samples and genetic information and for consenting to their use in research studies. We thank Tom Strachan (Newcastle) and Matt Deardorff and Ian Krantz (Children's Hospital of Philadelphia) for their gifts of CdLS LCLs. We thank the Institute of Genetics and Cancer (IGC) Advanced Imaging Resource for assistance with imaging and Ilya 
Fliamer for his help with deposition of data on GEO. We are indebted to Iain Williamson and Shelagh Boyle (IGMM) for mESC karyotyping. We also thank the ImagoSeine core facility (Institut Jacques Monod, France-BioImaging ANR-10-INBS-04) for confocal microscopy and the imaging platform of the Epigenetics and Cell Fate Center for epifluorescence microscopy for the micro-irradiation experiments. We thank Andrew Jackson (IGMM, Edinburgh) for feedback on the manuscript. We thank Valérie Doye (IJM, Paris) for advice during the revisions of the manuscript. G.O. was supported by a PhD studentship from the Medical Research Council (MRC). M.M.P. is supported by the UKRI/MRC grant (MR/T000783/1) and Barts charity (MGU0475) grants. D.R.F is supported by MRC University Unit grant (MC_UU_00007/3) and by the Simons Initiative for the Developing Brain. W.A.B. is supported by MRC University Unit grant (MC_UU_00007/2). C.B. was supported by a H2020 Marie-Curie Individual Fellowship (655350-NPCChr), a Bettencourt-Schueller foundation prize for young researchers, a grant from the Fondation ARC (Projet Fondation ARC PJA 20191209584) and a grant from the LIGUE contre le cancer île de France (RS20/75-53). S.P. and S.E.P. are supported by the French National Research Agency (ANR-18-CE12-0017-01). S.E.P. is an EMBO Young Investigator.

\section{Author contributions}

W.A.B., M.M.P. and C.B. conceived and designed the experiments with input from D.R. F. G.O. conducted most of the experiments with help from M.M.P. for ChIPseq and RNAseq experiments. C.B. performed immunostainings and analysis of RIF1 and MAD2L2. G.R.G. provided bioinformatic analysis of expression data. S.E.P. and S.P performed the laser micro-irradiation experiment. G.O., W.A.B. and C.B. wrote the manuscript with input from all authors.

\section{Competing interests}

The authors declare no competing interests.

\section{Additional information}

Supplementary information The online version contains supplementary material available at https://doi.org/10.1038/s41467-021-23500-6.

Correspondence and requests for materials should be addressed to W.A.B. or C.B.

Peer review information Nature Communications thanks the anonymous reviewer(s) for their contribution to the peer review of this work.

Reprints and permission information is available at http://www.nature.com/reprints

Publisher's note Springer Nature remains neutral with regard to jurisdictional claims in published maps and institutional affiliations.

(c) Open Access This article is licensed under a Creative Commons Attribution 4.0 International License, which permits use, sharing, adaptation, distribution and reproduction in any medium or format, as long as you give appropriate credit to the original author(s) and the source, provide a link to the Creative Commons license, and indicate if changes were made. The images or other third party material in this article are included in the article's Creative Commons license, unless indicated otherwise in a credit line to the material. If material is not included in the article's Creative Commons license and your intended use is not permitted by statutory regulation or exceeds the permitted use, you will need to obtain permission directly from the copyright holder. To view a copy of this license, visit http://creativecommons.org/ licenses/by/4.0/.

(C) The Author(s) 2021 